\title{
Highly r-process enhanced stars: New perspectives on old stars
}

\author{
Timothy C. Beers ${ }^{1}$ \\ National Optical Astronomy Observatory, Tucson, USA \\ E-mail:beers@noao.edu
}

I summarize the observational approach used to discover the majority of $r$-process-enhanced stars that have been discovered to date, and describe new efforts to identify additional representatives of this class, based on ongoing and future large-scale surveys. I comment on the implications of a recently discovered extremely metal-poor main sequence star with large $r$-process enhancement, a set of metal-poor stars with measured abundances of Tellurium, and the progress of an ongoing program to put limits on the binary properties of such stars, which is an important missing piece of information concerning the nature of the $r$-process itself.

XII International Symposium on Nuclei in the Cosmos August 5-12, 2012

Cairns, Australia

\footnotetext{
$1 \quad$ Speaker
} 


\section{Introduction}

In the "olden times" (for argument's sake, the pre-1980's), the only astrophysical objects available for comparison of the observed elemental and isotopic abundance patterns with the theoretical predictions of the $s$-process and the $r$-process were the Sun, a small number of meteorites, and the handful of metal-poor $([\mathrm{Fe} / \mathrm{H}]<-1.0)$ and very metal-poor $([\mathrm{Fe} / \mathrm{H}]<-2.0)$ stars that were known at the time. Although [1] and [2] stated the essential ideas associated with the astrophysical processes (and possible sites) needed in order to account for the production of the elements beyond $\mathrm{H}$ and $\mathrm{He}$, this was of course only the beginning of the search for understanding. What was required was a substantial expansion in the numbers of stars of lower metallicity, in particular the very metal-poor stars, since they are expected to have sampled the chemistry of the early Universe. It is these stars that enable the identification of the (relatively) rare examples of neutron-capture-element enhanced stars that provide crucial information about the astrophysical site(s) of the $s$-process and $r$-process.

Over the course of the past several decades, ever larger and more comprehensive efforts have been made to carry out dedicated surveys for stars of the lowest metallicity, such as the HK Survey of Beers and colleagues [3,4] and the Hamburg/ESO Survey (HES) of Christlieb and colleagues [5], culminating with the modern mega-surveys such as SDSS [6,7]. In a separate contribution to this volume, Beers \& Lee describe the development, calibration, and implementation of a software pipeline (the SEGUE Stellar Parameter Pipeline: SSPP) that is capable of estimating the stellar physical parameters, such as effective temperature, $T_{\text {eff }}$, surface gravity, $\log g$, and metallicity, $[\mathrm{Fe} / \mathrm{H}]$, in a robust and efficient manner for the large numbers of available medium-resolution $(R \sim 2000)$ SDSS/SEGUE spectra. Table 1 provides an illustration of how far we have progressed, by comparison of the numbers of stars with ever lower metallicity that were known before and after the SDSS/SEGUE program.

\begin{tabular}{lccc}
\multicolumn{1}{c}{ Class Name } & Metallicity Range & Pre-SDSS/SEGUE & Post-SDSS/SEGUE \\
\hline Metal Poor & {$[\mathrm{Fe} / \mathrm{H}]<-1.0$} & 15,000 & $150,000+$ \\
Very Metal Poor & {$[\mathrm{Fe} / \mathrm{H}]<-2.0$} & 3,000 & $30,000+$ \\
Extremely Metal Poor & {$[\mathrm{Fe} / \mathrm{H}]<-3.0$} & 400 & $1000+$ \\
Ultra Metal Poor & {$[\mathrm{Fe} / \mathrm{H}]<-4.0$} & 5 & 8 \\
Hyper Metal Poor & {$[\mathrm{Fe} / \mathrm{H}]<-5.0$} & 2 & 3 \\
Mega Metal Poor & {$[\mathrm{Fe} / \mathrm{H}]<-6.0$} & 0 & 0 \\
& & & \\
\hline
\end{tabular}

Table 1: Metal-poor stars in various levels of decreasing $[\mathrm{Fe} / \mathrm{H}]$. The final two columns indicate the numbers of stars known in each interval before and after the SDSS/SEGUE surveys, respectively. 
It is worth noting that, in Table 1 , the numbers of stars listed with $[\mathrm{Fe} / \mathrm{H}]<-4.0,[\mathrm{Fe} / \mathrm{H}]$ $<-5.0$, and perhaps even $[\mathrm{Fe} / \mathrm{H}]<-6.0$ are likely to increase in the near future, as highresolution spectroscopy is obtained for these objects. At medium spectral resolution, estimates of $[\mathrm{Fe} / \mathrm{H}]$ for individual stars of the lowest metallicity are hampered by the presence of interstellar $\mathrm{Ca}$ II and/or molecular carbon lines, perturbing the derived metallicity.

Once large numbers of candidate metal-poor stars are obtained, one generally requires highresolution spectroscopy in order to identify the characteristic elemental abundance patterns that can be used to associate the progenitor(s) of a given star with the likely astrophysical nucleosynthetic processes involved. In this brief summary, I concentrate on the highly $r$ process-enhanced stars, noted as $r$-II in Table 2.

Class Name Abundance Requirements

$\begin{array}{ll}r \text {-I } & +0.3 \leq[\mathrm{Eu} / \mathrm{Fe}] \leq+1.0 \text { and }[\mathrm{Ba} / \mathrm{Eu}]<0 \\ r \text {-II } & {[\mathrm{Eu} / \mathrm{Fe}]>+1.0 \text { and }[\mathrm{Ba} / \mathrm{Eu}]<0} \\ s & {[\mathrm{Ba} / \mathrm{Fe}]>+1.0 \text { and }[\mathrm{Ba} / \mathrm{Eu}]>+0.5} \\ r / s & 0.0<[\mathrm{Ba} / \mathrm{Eu}]<+0.5\end{array}$

Table 2: Classes of neutron-capture-element rich metal-poor stars, and their adopted limits on various abundance ratios. Adapted from [8].

\section{Searches for r-II stars}

As can be seen in Table 2, confident identification of $r$-II stars requires estimates for both the $[\mathrm{Eu} / \mathrm{Fe}]$ and $[\mathrm{Ba} / \mathrm{Eu}]$ abundance ratios, which can only be obtained from high-resolution spectroscopy; for stars fainter than about $14^{\text {th }}$ magnitude, such data requires the use of highthroughput spectrographs on 8-m class telescopes. Only one large survey, specifically designed for the detection of $r$-II stars, has been carried out to date, the HERES survey described by $[9,10]$. As a result of this survey, we know that the fraction of $r$-II stars among the very metalpoor stars is roughly 5\%. Already from the results of the HERES survey, it is clear that there exists a strong metallicity dependence for the detection of $r$-I (moderately r-process-enhanced stars) and the highly-enhanced $r$-II stars, as seen in Figure 1. Clearly, although $r$-I stars can be found over a broad range of metallicity, the $r$-II stars cluster in the metallicity range $-3.5<[\mathrm{Fe} / \mathrm{H}]<-2.5$. Serendipitous identifications of $r$-II stars in the past few years have identified a handful of additional $r$-II stars, so that today on the order of 18 such stars are known. Future, more directed searches (e.g., emphasizing the pertinent metallicity regime) are clearly desirable for increasing the number of known $r$-II stars. 

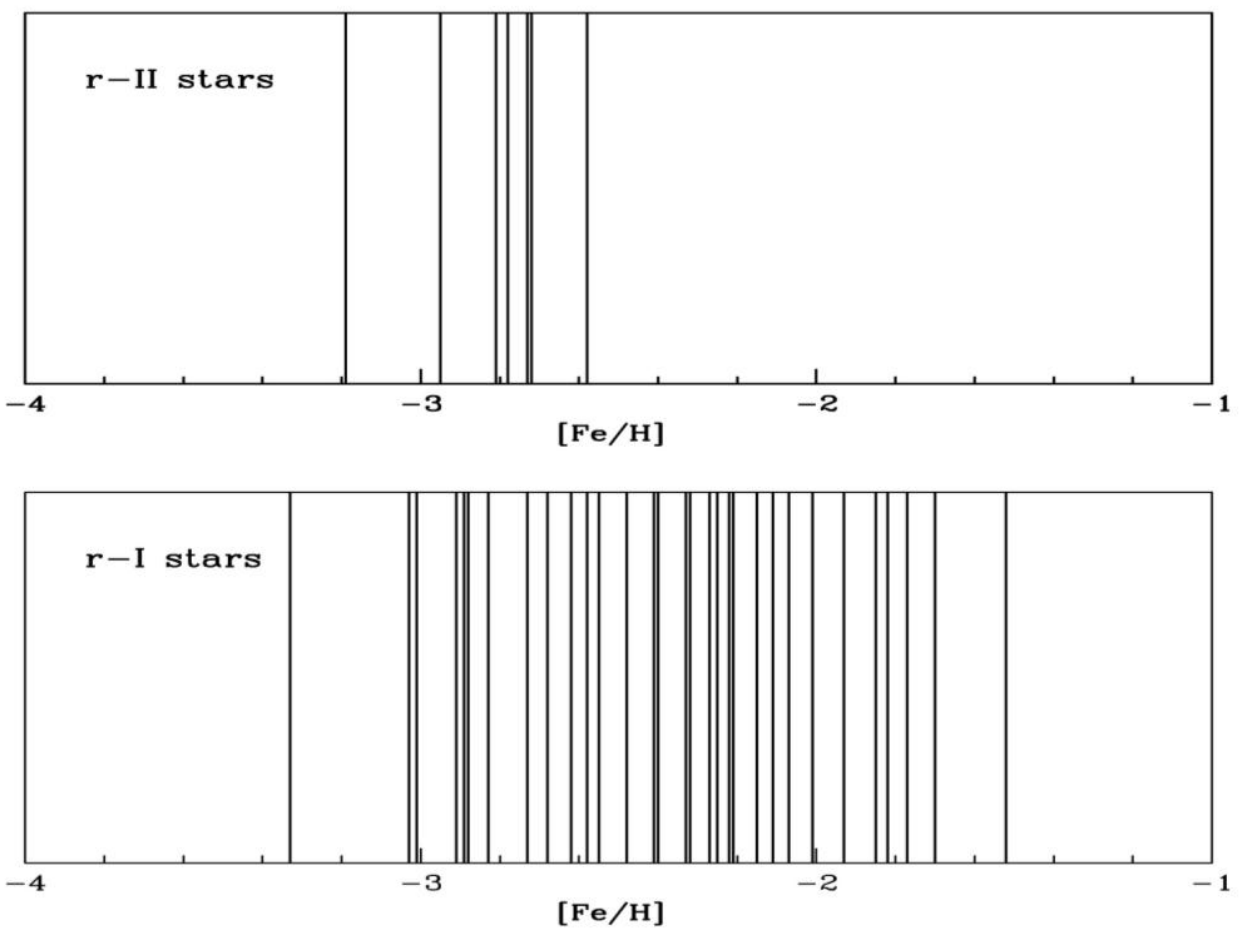

Figure 1: Distribution of $[\mathrm{Fe} / \mathrm{H}]$ for stars classified as $r$-II (top panel) or $r$-I from the HERES survey. Note the relatively narrow range over which $r$-II stars are found compared to the $r$-I stars.

\section{Observational progress on the nature of the r-process}

Space limitations preclude a complete discussion of recent observational progress, hence we highly three recent results, some of which are dealt with in more detail in other contributions to this volume. These are (1) The recent identification of a main-sequence $r$-II star, (2) The measurement of the tellurium (Te) abundance for a sample of metal-poor stars, and (3) The demonstration that binarity is not required for the production of $r$-I and $r$-II stars.

\subsection{A main-sequence $r$-II star}

The star SDSS J2357-0052 was shown recently to be a cool $\left(T_{\text {eff }} \sim 5000 \mathrm{~K}\right)$ main-sequence dwarf with $[\mathrm{Fe} / \mathrm{H}]=-3.4$, and $[\mathrm{Eu} / \mathrm{Fe}]=+2.0$, making it simultaneously the lowest metallicity and most Eu-enriched $r$-II star yet found [11]. This star is of particular interest, since all previous $r$-II stars identified to date have been in their giant-branch stage of evolution. Since dwarfs of the temperature of SDSS J2357-0052 do not have convective atmospheres, it can be reasonably concluded that the $r$-process-enhancement phenomenon is not due to some chemical peculiarity arising from the presence of a convective envelope in such stars. Unless binarity is involved, which we argue below is unlikely to be the case, this star was born from gas that had been previously polluted by one or more progenitors capable of producing the observed $r$-process abundance pattern. It follows that SDSS J2357-0052 is $r$-process-element enhanced through and through. 

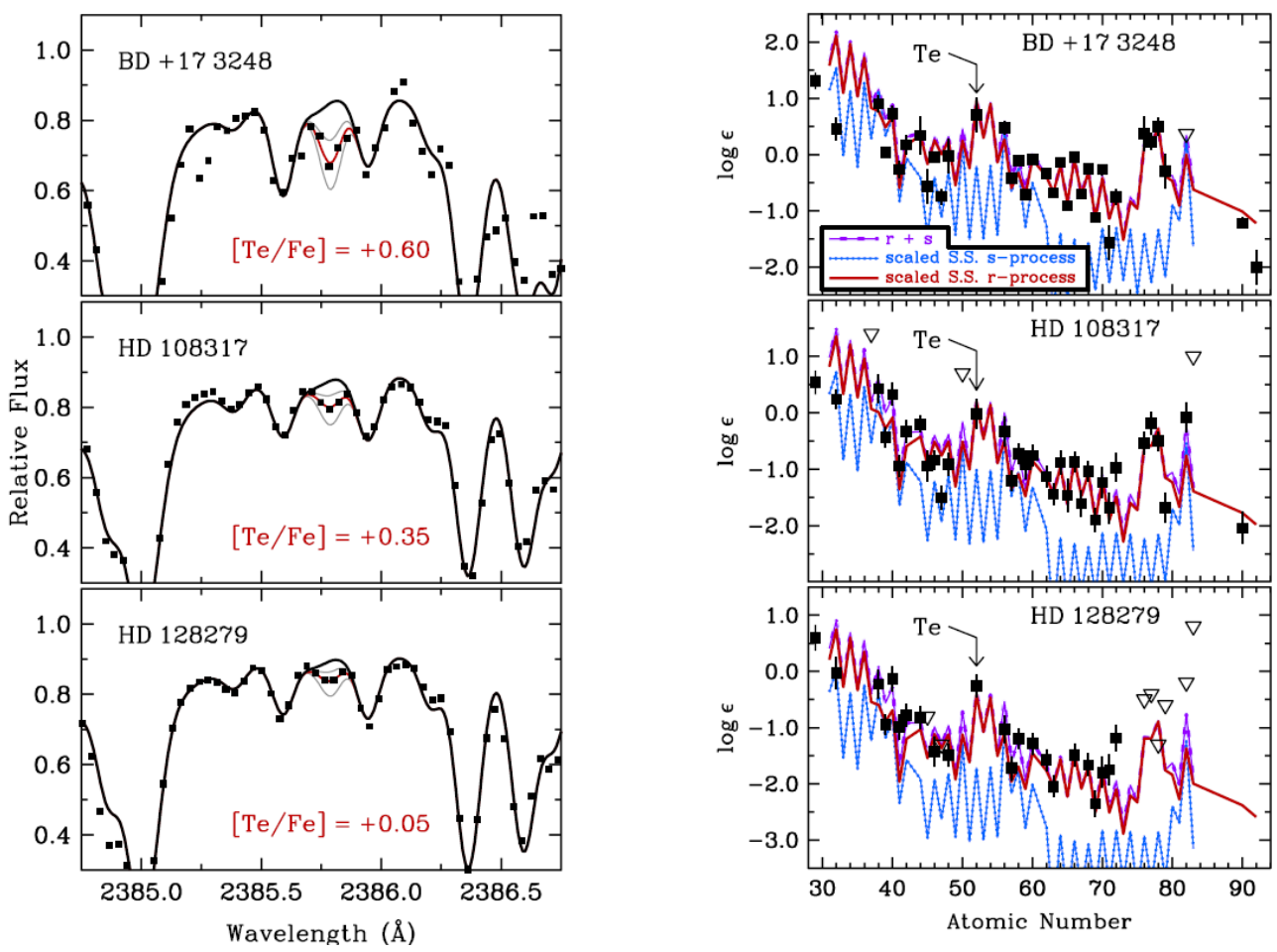

Figure 2: Detections of tellurium in near-UV spectra of three metal-poor stars with STIS on the Hubble Space Telescope. The left column of panels show the discovery spectra in the region of the Te feature, along with the derived $[\mathrm{Te} / \mathrm{Fe}]$ ratios. The right column of panels show the location of the detected Te on the $2^{\text {nd }}$ r-process peak. Figure taken from [12].

\subsection{Measurement of a Te abundance for metal-poor stars}

Elements near the so-called $2^{\text {nd }} r$-process peak have proven notoriously difficult to detect and measure, due to the need to obtain high-resolution spectroscopy of bright metal-poor stars (in order to obtain the required $\mathrm{S} / \mathrm{N}$ ) in the near-ultraviolet, with the only instrument currently capable of such observations, STIS on the Hubble Space Telescope. This barrier has recently been broken, as [12] detected neutral tellurium in three metal-poor stars enriched by products of $r$-process nucleosynthesis, BD+17 3248, HD 108317, and HD 128279. Tellurium ( $Z=52)$ is found at the $2^{\text {nd }}$ r-process peak $(A=130)$ associated with the $\mathrm{N}=82$ neutron shell closure, and it has not been detected previously in Galactic halo stars. The derived tellurium abundances match the scaled solar system $r$-process distribution to within the uncertainties, confirming the predicted second peak $r$-process residuals. These results suggest that tellurium is predominantly produced in the main component of the $r$-process, along with the rare-earth elements. Unfortunately, further exploration of elements in this region will likely not be possible once HST/STIS is no longer available. 


\subsection{The binary nature of $r$-I and $r$-II stars}

Ever since their discovery, it has been speculated that the enhancements of $r$-process elements in $r$-I and $r$-II stars may be due to their exposure to either direct Roche-lobe overflow, or the accretion of winds from binary companions responsible for the production of the $r$-process elements now observed on the surviving star. This possibility has been recently put to the test [13] (also described elsewhere in this volume). These authors report results obtained from four years of monitoring the radial velocities for $17 r$-process-enhanced $r$-I and $r$-II giants, in order to detect and characterize binaries in this sample. Their primary findings:

- Detection of three (possibly four) spectroscopic binaries with orbital periods and eccentricities that are indistinguishable from those of Population I binaries with giant primaries

- Detected binaries exhibit no signs that the secondary components have passed through the asymptotic giant-branch stage of evolution or exploded as supernovae. The other 14 stars in the sample appear to be single stars.

- Conclusion: The $r$-process enhancement of these stars was not a local event due to mass transfer or winds from a binary companion, but was imprinted on the natal molecular clouds of these (single and binary) stars by an external source.

Of particular interest is the reported result for the canonical $r$-II star, CS 22892-052, for which previous radial velocity monitoring allowed the possibility of a binary system, with a peak-to-peak variation on the order of $2 \mathrm{~km} / \mathrm{s}$ [14]. As shown in Figure 3, the higher accuracy measurements reported by [13] appear to eliminate this possibility, although future observations are surely warrented.

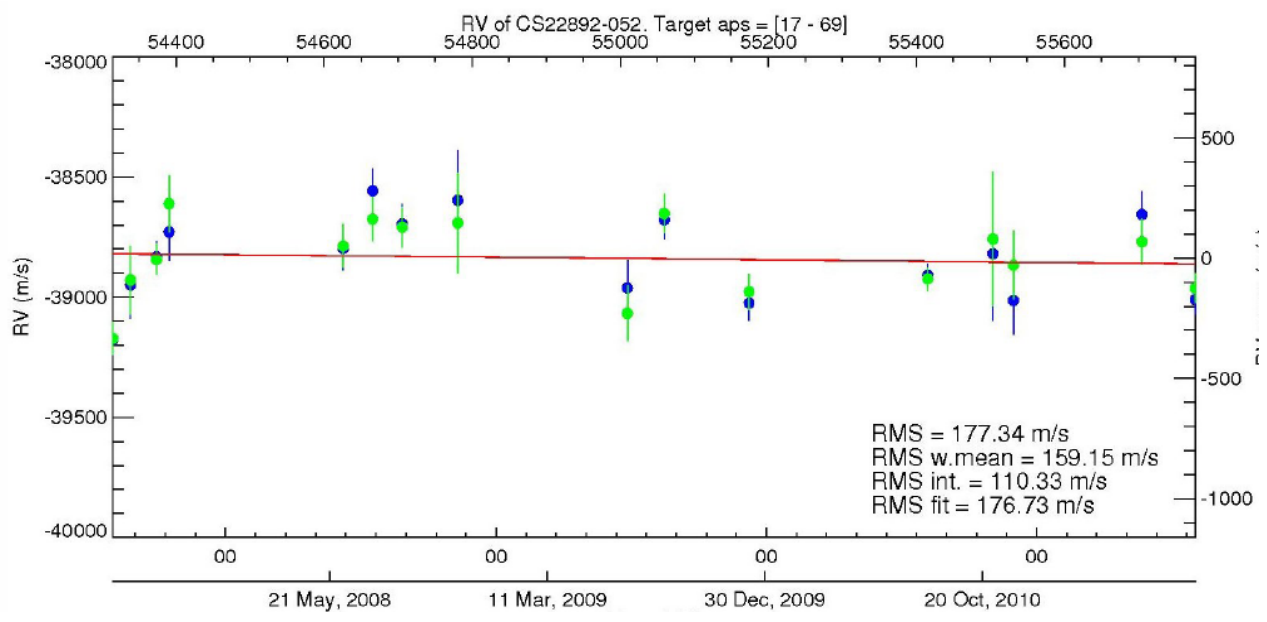

Figure 3: Radial velocity measurements of CS 22892-052 from [13], conducted with the Echelle spectrograph on the Nordic Optical Telescope. Note that the reported rms variations, on the order of 160 $\mathrm{m} / \mathrm{s}$ relative to the mean velocity, exclude the larger variations reported by [14]. 


\section{Surveys for the future}

In spite of the discovery of the r-process enhancement phenomenon over a decade ago, less than 20 examples of $r$-II stars are presently known. This is primarily due to the requirement of high-resolution spectroscopic follow-up of very metal-poor stars with 8-m class telescopes, in order to identify such stars. A renewed effort is called for, aimed at exploring the metallicity regime now known to favor the detection of $r$-II stars $(-3.5<[\mathrm{Fe} / \mathrm{H}]<-2.5)$, based on the many hundreds of such stars revealed from SDSS/SEGUE in the optimal temperature range for detection of $r$-process elements such as $\mathrm{Eu}\left(4500 \mathrm{~K}<T_{\text {eff }}<5250 \mathrm{~K}\right)$. In addition, long-term radial velocity monitoring of known $r$-I and $r$-II stars need to be executed, as this will place more stringent limits on the likelihood that binarity may (or may not) play a role in their origin. need to be carried out. Both kinds of studies are now feasible, with existing telescopes and instrumentation, if sufficient telescope time is granted. Certainly, the opportunity to better understand the nature of the $r$-process offers the justification for such efforts.

Numerous medium-resolution spectroscopic survey efforts are in the process of execution, or are planned for the near future. These large-scale surveys will further increase the chance to populate input lists for high-resolution spectroscopic follow-up dedicated to finding yet more examples of the r-process-element enhancement phenomenon. In this way, one can expect that the entire range of behavior of this unique class of stars will be explored. Surprises are to be expected!

\section{References}

[1] E. M. Burbidge et al., Synthesis of Elements in the Stars, Rev. Mod. Physics 1957 (29) 547-650.

[2] A. G. W. Cameron, Nuclear Reactions in Stars and Nucleogenesis, PASP 1957 (69) 201-222.

[3] T. C. Beers, G. W. Preston, \& S. A. Shectman, A Search for Stars of Very Low Metal Abundance. I., AJ 1985 (90) 2089-2102.

[4] T. C. Beers, G. W. Preston, \& S. A. Shectman, , A Search for Stars of Very Low Metal Abundance. II., AJ 1992 (103) 1987-2034.

[5] N. Christlieb, Finding the Most Metal-Poor Stars of the Galactic Halo with the Hamburg/ESO Objective-Prism Survey, Rev. Mod. Astron. 2003 (16) 191-206.

[6] D. G. York et al., The Sloan Digital Sky Survey: Technical Summary, AJ 2000 (120) 1579-1587.

[7] Z. Ivezic, T. C. Beers, \& M. Juric, Galactic Stellar Populations in the Era of the Sloan Digital Sky Survey and Other Large Surveys, Ann. Rev. A. \& A. 2012 (50) 251-304.

[8] T. C. Beers \& N. Christlieb, The Discovery and Analysis of Very Metal-Poor Stars in the Galaxy, Ann. Rev. A. \& A. 2005 (43) 531-580.

[9] N. Christlieb et al., The Hamburg/ESO R-process Enhanced Star Survey (HERES). I. Project Description, and Discovery of Two Stars with Strong Enhancements of Neutron-capture Elements, A \& A 2004 (428) 1027-1047. 
[10] P. S. Barklem et al., The Hamburg/ESO R-process Enhanced Star Survey (HERES). II.

Spectroscopic Analysis of the Survey Sample, A \& A 2005 (439) 129-151.

[11] W. Aoki et al., Extreme Enhancements of r-process Elements in the Cool Metal-poor Mainsequence Star SDSS J2357-0052, ApJ 2010 (723) L201-L206.

[12] I. U. Roederer et al., Detection of the Second r-process Peak Element Tellurium in Metal-poor Stars, ApJ 2012 (747) L8.

[13] T. Hansen et al., The Binary Frequency of r-Process-element-enhanced Metal-poor Stars and Its Implication: Chemical Tagging in the Primative Halo of the Milky Way, ApJ 2001 (743) L1.

[14] G. W. Preston \& A. Sneden, The Incidence of Binaries among Very Metal-poor Carbon Stars, AJ 2001 (122) 1545-1560. 